\title{
Parental Attitude to Influenza Infection: Willingness for Childhood Vaccination
}

Yuxin Woon ${ }^{1^{*}}$ and Edina Moylett ${ }^{1,2}$

${ }^{1}$ School of Medicine, National University of Ireland, Galway, Ireland

${ }^{2}$ Academic Department of Paediatrics, National University of Ireland, Galway, Ireland

\begin{abstract}
Introduction: As the 2020- 2021 flu season coincides with the currently circulating Coronavirus disease 2019 (COVID-19), Ireland is introducing free influenza vaccines to children ages $2-12$ this year. This is to minimise the disease and economic burden of influenza to an already- strained hospital system due to the ongoing pandemic. Therefore, it is important to identify factors that may affect vaccine uptake.
\end{abstract}

Objective: To explore parental knowledge and attitudes towards influenza infection and potential factors affecting willingness to routinely vaccinate their child (ren).

Methods: This descriptive study involved interviewing parents $(n=300)$ attending the paediatric outpatient department (OPD) at University Hospital Galway. A pilot study assisted with standardizing the questionnaire and in optimizing the clinical catchment area (Emergency Department vs. postnatal ward vs. OPD clinics). Galway Clinical Research Ethics Committee granted the ethical approval and data was analyzed using SPSS.

Results: The majority of respondents were Irish (251, 83.7\%), 236 (78.7\%) with private health insurance. The commonest age range was $31-40$ years $(163,54.3 \%)$. Less than $40 \%$ had a Bachelor's degree (113, $37.7 \%)$ as their highest education level. Most participants $(226,75.3 \%)$ agreed with annual influenza vaccine for their child if recommended.

The following factors were shown to positively affect potential annual influenza vaccine uptake $(\mathrm{p}<0.05)$ : Positive general perception towards childhood immunization $(217,96.1 \%)$

Parents who received the influenza vaccine $(127,56.2 \%)$

Mothers who received the influenza vaccine antenatally $(81,42.4 \%)$

Positive childhood immunization experiences $(223,98.7 \%)$

Amongst community supporting influenza vaccination $(167,73.9 \%)$

No concerns about influenza vaccine $(200,88.5 \%)$

High test score for parental knowledge on influenza vaccine $(33.6 \%, \mathrm{~N}=76$, achieved full marks, mean score $=4.57 \pm 1.28$ ).

Conclusion: The overall feedback for routine paediatric influenza vaccination was positive. Parental knowledge, attitudes, prior history of vaccination and social norms each had an independent influence on parents' willingness to vaccinate their child. A general lack of awareness of paediatric influenza immunization was highlighted and demonstrates the need to improve immunization awareness strategies.

\section{Introduction}

The 2020-2021 flu season will coincide with the currently circulating Coronavirus disease 2019 (COVID-19), which shares similar symptoms with the seasonal influenza [1]. Influenza is a highly infectious, acute viral respiratory tract infection which can cause severe or even fatal complications in children, especially in infants below 6 months [2]. Australia, which has had its flu season as COVID-19 started to spread, reported a record low flu season of 21,187 cases as compared to 298,120 in 2019 [3,4]. This can be attributed to their increased influenza vaccine uptake in addition to the pandemic precautions such as social distancing and wearing face masks in public. 18 million vaccinations were given this flu season as compared to 13 million last year [5]. Therefore, it is crucial to encourage influenza vaccine uptake to minimise the pressure on an already- strained hospital system.

In May 2020, Ireland has announced that the influenza vaccine will be free for all children aged 2 to 12 years old for the upcoming flu season [6]. While this is the first time the vaccine is being offered to healthy children, it has been provided to children with underlying health conditions for several years. In fact, the introduction of routine paediatric influenza vaccination has been anticipated prior to this global pandemic.

One of the reasons is the increased severity and prevalence of influenza amongst the paediatric population in recent years. A report from University Hospital Galway (UHG) highlighted an increase in influenza cases of $350 \%$ between the 2014 and 2016 'influenza seasons'. The potential for severe influenza related complications among the paediatric age group was also highlighted, $7 \%$ of those hospitalised developed severe complications with the death of one child with underlying global developmental delay. None of those admitted had received influenza vaccine despite being in defined 'at risk' groups [7].

*Corresponding Author: Dr. Yuxin Woon, Academic Department of Paediatrics, Clinical Science Institute, National University of Ireland, Galway, Ireland, Tel: +353 83 8889359; E-mail: yuxinwoon@gmail.com

Citation: Woon Y, Moylett E (2020) Parental Attitude to Influenza Infection: Willingness for Childhood Vaccination. J Pediatr Neonat Care 6: 169. doi: https:// doi.org/10.15344/2455-2364/2020/169

Copyright: (C) 2020 Woon et al. This is an open-access article distributed under the terms of the Creative Commons Attribution License, which permits unrestricted use, distribution, and reproduction in any medium, provided the original author and source are credited. 
Aside from the clinical burden, influenza infers substantial economic burden related to hospital admission, physician visits and absenteeism among both children and their parents [8].

Children are often the primary vector in the transmission of influenza infection. Universal childhood influenza vaccination not only benefits the vaccinated children, but also provides indirect protection (herd immunity) to family members and community. Recently Bambury et al. reported that vaccinating children against influenza has a greater potential for reducing total influenza deaths in comparison to vaccination strategies that target solely the elderly population or healthcare professionals [9]. The latter is further supported by a reduction in disease burden of seasonal influenza since the UK extended its vaccination programme to all 2 to 3 year olds. There was a $6.2-9.9 \%$ reduction in general practitioner visits due to influenza- like- illness in the general population, $6.1-10.7 \%$ reduction in influenza-attributable respiratory hospitalisations and a $0.9-2.4 \%$ reduction in overall respiratory deaths [10].

While some countries have routinely vaccinated those more atrisk of influenza complications, countries like the USA, UK, Canada and Finland have begun to recommend mass vaccination among the paediatric population in the last 10 years [11]. In 2016, the Joint Committee on Vaccination and Immunisation (JCVI) reviewed childhood influenza immunisation programmes across countries which demonstrated good overall cost-effectiveness and recommends the continuation of routine seasonal childhood influenza vaccination [12].

As Ireland prepares to introduce the universal annual paediatric influenza vaccine for the first time this year, it is crucial to identify key factors affecting routine influenza vaccination uptake. Previous studies have highlighted that parental views have a major role in vaccine uptake as they ultimately decide whether their children should be vaccinated [13]. Unfortunately, there is limited literature on Irish parental views on childhood immunisations. Therefore, the aim of this study was to explore parents' perspective on influenza vaccine and their acceptability towards the introduction of annual paediatric influenza vaccine. This would provide a framework of issues to be addressed which would assist constructing an effective immunization program.

\section{Methods}

Descriptive, cross-sectional study involving 300 parents (not both parents) interviewed by a single trained researcher at the paediatric Out Patient Department (OPD) at UHG. The interviews were conducted based on a standardized questionnaire with reference to an initial pilot study completed in the same hospital. In addition, the pilot study identified the paediatric OPD as the optimal clinical catchment area.

The study was conducted during May to June 2018, each interview took approximately 10 minutes to complete. A convenience sampling method was applied, and respondents were invited to participate in the study while awaiting paediatric consultation.

Basic demographic and socioeconomic information were elicited through multiple-choice questioning. Respondents' knowledge on influenza was tested in a series of 6 questions and given a test score. Both Likert scales and 'yes/no' questions were used, for example, 'the influenza vaccine can cause influenza, do you strongly agree, agree, don't know, disagree, strongly disagree?' and 'can influenza be lifethreatening?'

Another series of questions were designed with reference to the Health Belief Model (HBM) and other factors suggested by literature to understand parental perceptions on influenza vaccine. The HBM is a widely used psychological health behaviour change model developed to predict health- related behaviours [14]. Similarly, both Likert scales and 'yes/no' questions were applied, for example, 'has your child ever received the influenza vaccine?' Parental attitudes and beliefs were recorded descriptively and recategorized, for example, 'do you have a specific concern for the influenza vaccine? If yes, what is it?' The results were later subdivided into the necessity, efficacy, side effects of the influenza vaccine etc. Answers were compared to their willingness to routinely vaccinate their child with the influenza vaccine, should it be recommended along with routine childhood vaccinations. This was measured using a 'yes/ no' question.

The study was approved by the Galway Clinical Research Ethics Committee and verbal informed consent was obtained from all individual participants included in the study prior to commencing the interviews.

\section{Data analysis}

Independent variables were divided into $\mathrm{HBM}$ variables and others that the researchers regarded to be relevant to the study. The dependent variable was parental willingness to routinely vaccinate their children against the influenza infection. Chi-square was used on these variables. For continuous data, Shapiro-Wilk test of normality was performed. All data were analysed using IBM SPSS version 23 and a p-value of $<0.05$ was taken as statistically significant with a $95 \%$ confidence interval.

\section{Results}

A total of 373 parents were invited to partake and recruitment ceased once the target of 300 respondents was reached, giving a response rate of $80.4 \%$. Of those not included, 51 were unable to complete the interview owing to time constraints and 22 were unwilling to participate in the interview.

\section{Modifying variables}

\section{Demographics}

More than half of the respondents were aged 31 to 40 (163, 54.3\%), $105(35 \%)$ were 41 years and above, and $32(10.7 \%)$ were 30 years or below. Most were working, full-time (140, 46.7\%), part-time $(48,16 \%)$ or self-employed $(22,7.3 \%) .87$ respondents $(29 \%)$ were homemakers, $2(0.7 \%)$ were students and 1 parent $(0.3 \%)$ was a retiree. The respondents had a median of 2 children (minimum, 1; maximum, 9). A clear majority were married $(237,79 \%)$ and of Irish descent $(251,83.7 \%)$. The most common educational level achieved was a Bachelor's degree $(113,37.7 \%)$, followed by secondary school certificate $(78,26 \%)$, diploma $(52,17.3 \%)$, Master's degree $(49,16.3 \%)$ and a primary school certificate $(6,2 \%) ; 1$ respondent $(0.3 \%)$ had a $\mathrm{PhD}, 1$ without any formal education (0.3\%). A total of $236(78.7 \%)$ respondents had insurance coverage; $126(42 \%)$ had private insurance and $110(36.7 \%)$ had a medical card. 
Citation: Woon Y, Moylett E (2020) Parental Attitude to Influenza Infection: Willingness for Childhood Vaccination. J Pediatr Neonat Care 6: 169. doi: https:// doi.org/10.15344/2455-2364/2020/169

Page 3 of 5

\section{Parental influenza knowledge}

Respondents were given a maximum score of 6 based on a series of 6 questions designed to test their influenza knowledge. Of all respondents, only $75(25 \%)$ were aware that influenza could be lifethreatening and $56(18.7 \%)$ knew how frequently people should receive influenza vaccination. Most 'Agree' $(181,60.3 \%)$ or 'Strongly agree' $(34,11.3 \%)$ that annual influenza vaccine was effective at preventing influenza infection. The vast majority (289, 96.3\%) were aware children could develop influenza infection. In regard to influenza vaccine side-effects, 191 (63.7\%) respondents were able to name a known side effect while $86(28.7 \%)$ were unaware of any side effects. Of those who answered incorrectly, answers included influenza infection $(8,34.8 \%)$, nausea $(6,26.1 \%)$, weakened immune system $(4,17.4 \%)$, sore throat $(3,13 \%)$ and death $(2,8.7 \%)$. Lastly, 61 $(20.3 \%)$ respondents perceived that the influenza vaccine could cause influenza infection.

The respondents displayed a moderate level of influenza knowledge, median score of 4 (minimum, 1 ; maximum, 6). Parents with a higher knowledge score for influenza vaccine were significantly more willing to annually vaccinate their children (mean rank 136 versus 59, $\mathrm{p}=$ $0.002)$.

\section{Social norms related to influenza vaccination}

A total of 201 (67\%) respondents reportedly were among friends and family who were generally supportive of the influenza vaccine. Of those among unsupportive friends and family (99, 33\%), specific reasons are related to vaccination side effects $(27,27.3 \%)$ and vaccine necessity $(26,26.3 \%)$.

\section{General perception towards vaccination}

A total of 130 (43.4\%) respondents 'Agreed' and 151 (50.3\%) 'Strongly agreed' that immunizations are given for the benefit of their child and the population.

\section{Parental history of influenza vaccination}

In total, $146(48.7 \%)$ respondents received prior influenza vaccine. Regarding antenatal influenza vaccine, 90 (30\%) were compliant. Of those non-compliant, $60(36.2 \%)$ respondents stated they were not offered the vaccine, 52 (31.9\%) were concerned about side effects and $43(25.9 \%)$ perceived the vaccine to be unnecessary.

\section{Child's history of influenza vaccination}

In total $260(86.7 \%)$ respondents' children had not received prior influenza vaccine. These respondents were mostly not offered the vaccine $(224,86.2 \%)$. Of the $40(13.3 \%)$ respondents who vaccinated their children, $39(97.5 \%)$ were due to healthcare professional recommendation, and $1(2.5 \%)$ was upon parental request.

\section{Prior vaccine experience}

The vast majority of parents $(287,95.7 \%)$ were satisfied with their child's previous vaccinations. Of those who were unsatisfied, 10 (77\%) stated side effects post- vaccination and $3(23 \%)$ perceived that there were too many childhood vaccinations.

\section{Prior experience with influenza affecting their children}

A total of $278(92.7 \%)$ respondents had no prior experience with influenza (confirmed with a positive test) affecting their children.

\section{Perceptions related to influenza vaccination among children}

\section{Perceived susceptibility of contracting influenza infection}

Most respondents perceived that their child was just 'as likely' (180, $60 \%)$ as the general population to contract influenza infection; 65 (21.7\%) perceived that it was 'unlikely' for their child to contract the infection and $11(3.7 \%)$ perceived that it was 'very unlikely'. However, among those who answered 'unlikely' and 'very unlikely', 7 (10.8\%) and $4(36.4 \%)$ respectively immunized their child against influenza.

\section{Perceived severity of influenza infection}

Most respondents perceived that there was a medium chance (127, $42.3 \%$ ) for a child to be hospitalized for influenza infection. This was followed by 95 (31.7\%) respondents who perceived that children had a low chance of being hospitalized for the infection.

\section{Perceived benefits of paediatric influenza vaccine}

This was demonstrated by the $2^{\text {nd }}$ question in the parental knowledge test, 215 (71.6\%) respondents regarded the annual influenza vaccine as potentially effective at preventing influenza infection.

\section{Perceived barriers to paediatric influenza vaccine}

In total, 249 (83\%) respondents did not have specific concerns regarding influenza vaccine. Of those who had concerns, potential side effects were most common $(23,45 \%)$ followed by vaccine efficacy $(18,35.3 \%)$ and necessity $(4,7.8 \%)$.

\section{Cue to action}

Most of the respondents did not have children with chronic health conditions $(208,69.3 \%)$. Of those who had chronic health problems, asthma $(43,47.2 \%)$ and neurodevelopmental conditions (22, 24.2\%) were the most common health issues; only 61 (20.3\%) of these respondents were recommended the influenza vaccine for their child by a healthcare provider.

\section{Parental willingness to vaccinate}

Should the influenza vaccine be added to the routine childhood vaccination schedule, 226 (75.3\%) respondents were happy for their children to be vaccinated (Table 1). In addition to the level of parental knowledge on influenza vaccine, other statistically significant factors include parental history of influenza vaccination, social norms, and concerns regarding the vaccine (Table 2).

\section{Discussion}

Concerning annual influenza vaccine, $75.3 \%$ of parents surveyed were willing to annually vaccinate their child. This is an encouraging finding suggesting potential for a high vaccination uptake should annual influenza be recommended, exceeding the UK's current vaccine uptake ambition of $65 \%$ [15]. In line with a recently conducted Irish epidemiological review of paediatric influenza in whom only 12 to $14 \%$ of high-risk hospitalized children had received influenza vaccine (personal communication); similarly, $13.3 \%$ of children in this report had been vaccinated.

In this study, factors that proved to affect parental willingness to routine influenza immunization highlighted a common issue: the lack 
Citation: Woon Y, Moylett E (2020) Parental Attitude to Influenza Infection: Willingness for Childhood Vaccination. J Pediatr Neonat Care 6: 169. doi: https:// doi.org/10.15344/2455-2364/2020/169

Page 4 of 5

of awareness towards the seasonal vaccine. While the respondents generally had a moderate level of understanding of influenza, some of the results were surprising. For example, a large proportion of respondents $(72 \%)$ did not believe influenza infection could be lifethreatening, $63.5 \%$ of parents who disagreed to routine childhood influenza vaccination thought it was unnecessary and only $18.7 \%$ of respondents knew how often the vaccine should be administered. Moreover, there is also the worrying misconception that influenza vaccine causes influenza infection among $20.3 \%$ of respondents.

Previous research established that inadequate parental vaccination knowledge negatively affects childhood vaccination uptake [16,17]. This study found similar results in that parents with a higher level of influenza knowledge (median $=4, \mathrm{p}=0.002$ ) and parents without specific concerns regarding the influenza vaccine $(88.5 \%, \mathrm{p}<0.001)$ were more likely to vaccinate their child against the disease. Therefore, strategies for spreading awareness about childhood influenza needs to be revised and improved in order to address parental concerns and clarify inaccurate beliefs. Respondents who were among friends and family who were supportive of the influenza vaccine $(73.9 \%, \mathrm{p}<.001)$ were also more likely to allow their children to receive the vaccine annually. This suggests that raising the awareness of both adult and paediatric influenza is warranted.

A generally positive perception towards vaccination (96.1\%, $\mathrm{p}=0.004$ ) leads to better vaccination uptake rate, which is in line with findings of a previous systematic review regarding vaccination uptake [18]. A positive past experience $(98.7 \%, \mathrm{p}<0.001)$ also encouraged parental intent to vaccinate. While little is known about family experiences during immunization visits, Stockwell et al. reported that negative vaccination experiences tend to result in under-immunized children [19]. Parents who have received the influenza vaccine (both in general $(56.2 \%, \mathrm{p}<0.001)$ and antenatally $(42.4 \%, \mathrm{p}<0.001)$ were found to encourage routine childhood influenza immunization as well. This ties in with the effect of positive social influence on parental intent to vaccinate. Health-seeking behaviors are often embedded in a family, and it is not surprising that parents who have received vaccinations would ensure their children were protected [20]. There

\begin{tabular}{|l|l|}
\hline Variables & $\mathrm{N}(\%)$ \\
\hline $\begin{array}{l}\text { If the influenza vaccine was to be administered with the routine childhood vaccinations, I would be } \\
\text { happy for my child to be vaccinated. }\end{array}$ & \\
\hline Yes & $226(75.3)$ \\
\hline No & $74(24.7)$ \\
\hline If no, why? & \\
\hline Unnecessary & $47(63.5)$ \\
\hline Side effects & $9(12.2)$ \\
\hline Insufficient knowledge & $9(12.2)$ \\
\hline Unsure of vaccine efficacy & $4(5.4)$ \\
\hline Inability to build up immunity & $5(6.7)$ \\
\hline
\end{tabular}

Table 1: Parental willingness to routinely vaccinate their child against influenza.

\begin{tabular}{|c|c|c|c|c|}
\hline Variables & Yes N (\%) & No $N(\%)$ & P-value & $95 \% \mathrm{CI}^{*}$ \\
\hline \multicolumn{5}{|c|}{ Parental history of influenza vaccination: } \\
\hline \multicolumn{5}{|c|}{ Have you ever received the influenza vaccination? } \\
\hline Yes & $127(56.2)$ & $19(25.7)$ & \multirow[t]{2}{*}{$<0.001$} & \multirow[t]{2}{*}{$0.43-0.54$} \\
\hline No & $99(43.8)$ & $55(74.3)$ & & \\
\hline \multicolumn{5}{|c|}{ Parental history of influenza vaccination: } \\
\hline \multicolumn{5}{|c|}{ Did you receive the influenza vaccine during your pregnancy? } \\
\hline Yes & $81(42.4)$ & $9(13.8)$ & \multirow[t]{2}{*}{$<0.001$} & \multirow[t]{2}{*}{$1.59-1.71$} \\
\hline No & $110(57.6)$ & $56(86.2)$ & & \\
\hline \multicolumn{5}{|c|}{ Social norm: } \\
\hline \multicolumn{5}{|c|}{ Among your friends and family, are they generally supportive of the influenza vaccine? } \\
\hline Yes & $167(73.9)$ & $35(47.3)$ & \multirow[t]{3}{*}{$<0.001$} & \multirow[t]{3}{*}{$0.34-0.58$} \\
\hline No & $52(23)$ & $38(51.4)$ & & \\
\hline Mixed & $7(3.1)$ & $1(1.4)$ & & \\
\hline \multicolumn{5}{|c|}{ Concerns on the influenza vaccine: } \\
\hline \multicolumn{5}{|c|}{ Do you have a specific concern for the influenza vaccine? } \\
\hline Yes & $26(11.5)$ & $25(33.8)$ & \multirow[t]{2}{*}{$<0.001$} & \multirow[t]{2}{*}{$2.20-2.30$} \\
\hline No & $200(88.5)$ & $49(66.2)$ & & \\
\hline
\end{tabular}

Table 2: Factors associated with parental willingness to routinely vaccinate.

$\mathrm{CI}^{*}=$ Confidence interval. 
Citation: Woon Y, Moylett E (2020) Parental Attitude to Influenza Infection: Willingness for Childhood Vaccination. J Pediatr Neonat Care 6: 169. doi: https:// doi.org/10.15344/2455-2364/2020/169

Page 5 of 5

was no association between demographics and parental willingness to routinely vaccinate a child against influenza.

\section{Limitations}

This study has several limitations. Firstly, the test used to determine parental knowledge on influenza was not a validated scale. Nonetheless, these questions were revised based on an initial pilot study. Secondly, due to the nature of the study, recall bias could have occurred as it involved past vaccination history and the data were not compared to the respective medical records. Selection bias could have occurred due to convenience sampling. Lastly, generalizing the results from this study with 300 respondents to the number of parents in Ireland is suboptimal.

\section{Conclusion}

The overall feedback for routine paediatric influenza vaccination was positive with $75.3 \%$ of respondents being supportive of its introduction. Good parental knowledge, positive attitudes towards immunizations, prior history of influenza vaccination, being in a pro-vaccination community and having no concerns regarding the influenza vaccine each independently encouraged parents to annually vaccinate their children against influenza infection. The key to ensure successful implementation of this vaccine would be to improve influenza awareness strategies among the general public. With reference to the UK's childhood influenza vaccination review, the Irish government can improve marketing campaigns through social media and the local press, increase engagement with all stakeholders including the children and parents as well as addressing specific misconceptions highlighted in this study [21].

With regard to future studies, it would be beneficial to explore the ranking of factors which affect the parental intent to vaccinate in order to highlight the more pressing issues that need to be addressed. Secondly, further studies can be done to explore the acceptability of the current intranasal vaccination versus the intramuscular alternative.

\section{Conflict of Interest}

The authors declare that they have no conflict of interest.

\section{Acknowledgements}

We thank the respondents for providing the useful information used in this study and Ms. Gloria Avalos for invaluable assistance with statistics.

\section{Funding}

This work received support from the School of Medicine Summer Scholarship, sponsored by the National University of Ireland, Galway.
3. Australian Government Department of Health (2020) Australian Influenza Surveillance Report.

4. Australian Government Department of Health (2019) Australian Influenza Surveillance Report.

5. Department of Health (2020) Record flu vaccines in 2020 to protect Australians.

6. Health Protection Surveillance Centre (2020) Influenza vaccines to be made available without charge to all children aged 2- 12.

7. Ryan G, Cleary A, Keady D, Allen N, Moylett E, et al. (2017) 2015- 2016 Influenza season in an Irish regional paediatric unit: importance of influenza vaccination highlighted. Irish Medical Journal 110: 609.

8. Fraaji PLA, Heikkinen T (2011) Seasonal influenza: The burden of disease in children. Vaccine 29: 7524- 7528.

9. Bambery B, Douglas T, Selgelid MJ, Maslen H, Giubilini A, et al. (2018) Influenza vaccination strategies should target children. Public Health Ethics 11: 221-234.

10. Rajaram S, Wiecek W, Lawson R, Blak B, Zhao Y, et al. (2017) Impact of increased influenza vaccination in 2- 3 year old children on disease burden within the general population: A Bayesian model- based approach. PLoS One 12: e0186739.

11. McGuire A, Drummond M, Keeping S (2016) Childhood and adolescent influenza vaccination in Europe: A review of current policies and recommendations for the future. Expert Rev Vaccines 15: 659-670.

12. Public Health England (2018) The National Childhood flu immunisation programme 2018/19: Information for healthcare practitioners. PHE.

13. Lind C, Russell ML, MacDonald J, Collins R, Frank C, et al. (2014) Schoolbased influenza vaccination: parents' perspectives. PLos One 9: e93490.

14. University of Twente. (2017) Health Belief Model. University of Twente.

15. Public Health England (2018) The national flu immunisation programme 2018/19. PHE.

16. Al- lela O, Bahari MB, Al- Qazaz HK, Salih M, Jamshed S, et al. (2014) Are parents' knowledge and practice regarding immunization related to pediatrics' immunization compliance? A mixed method study. BMC Paediatrics 14: 20.

17. Lau J, Mo P, Cai Y, Choi K (2013) Coverage and parental perceptions of influenza vaccination among parents of children aged 6 to 23 months in Hong Kong. BMC Public Health 13: 1026.

18. Smith L, Amlot R, Weinman J, Yiend J, Rubin G, et al. (2017) A systematic review of factors affecting vaccine uptake in young children. Vaccine 3 : 6059- 6069.

19. Stockwell M, Irigoyen M, Martinez R, Findley S (2011) How parents' negative experiences at immunization visits affect child immunization status in a community in New York City. Public Health Rep 126: 24- 32.

20. Greder KA, Sano Y (2011) Health- Seeking Behaviour in Families. Encyclopedia of Family Health. Human Development and Family Studies.

21. Kassianos G, White S, Reynolds AJ, Rajaram S (2015) Review of the experiences from the first childhood influenza vaccination programme with a live attenuated influenza vaccine in England and Scotland. Drugs Context 4: 212280 .

\section{References}

1. Grohskopf L, Alyanak E, Broder K, Fry A, Jernigan D, et al. (2020) Prevention and control of seasonal influenza with vaccines: recommendations of the Advisory Committee on Immunization Practices- United States, 2020-21 Influenza Season. MMWR Recomm Rep 69: 1-24.

2. Shang $M$, Blanton $L$, Brammer $L$, Olsen $S$, Fry $A$, et al. (2018) InfluenzaAssociated Pediatric deaths in the United States 2010- 2016. Pediatrics 141: 20172918. 